\title{
Results of 198 primary total hip arthroplasties using the Delta PF-FIT system with ceramic-on-ceramic articulating surfaces with average seven years follow \\ up
}

Petr Fulin", David Pokorny, Jan Hert and Antonin Sosna

\begin{abstract}
Background: The lifetime implants is a key parameter that the surgeon should take into account at the time of the primary total hip arthroplasty (THA). The aim of this study was a clinical and radiographical evaluation of the Delta PF-FIT (LimaCorporate, Italy) THA system with ceramic-on-ceramic articulations. We have not found a clinical or radiographical assessment of this implant in available published literature.

Methods: A total of $197(F=94, M=103)$ primary THAs were evaluated in 163 patients with a mean follow-up of 7.7 years (range 5.1-11.2 years (SD \pm 1.5$)$ ) Harris hip Score (HHS) and the Western Ontario and McMaster Universities Arthritis index (WOMAC) were used for the clinical evaluation. The statistical evaluation was processed by standard statistical methods. The study was approved by Ethic Committee of the University Hospital Motol (Reference No. EK-73/19).

Results: The mean HHS score was found to be 97.59 points (61-100 range with a \pm 5.13 SD, preoperative HSS was 51.21, range 28-73 with a $\pm 4,77$ SD). 186 THAs were evaluated as excellent (90-100 points), 9 THAs rated as good (80-89 points), 1 THA was rated as fair (70-79) points and 1 THA rated as poor (less than 70 points). The mean WOMAC score was 97.38 points (65-100 range with a \pm 5.18 SD, preoperative was 50,12, range 27-69 with a \pm 4.85 SD). We documented an overall $99.49 \%$ Kaplan-Meier survival with a mean follow-up of 7.7 years with the FIT (LimaCorporate) stem revision and any component revision as the endpoint. With the Delta PF (LimaCorporate) cup revision as the endpoint, the survival was 100\%. We have not found a previously published clinical or radiographical review of this THA system, the study shows a comparison with other THA implants.
\end{abstract}

Conclusion: Evaluation of the Delta-PF-FIT (LimaCorporate, Italy) THA system with the use of ceramic-on-ceramic BIOLOX Delta articulation surfaces shows very good outcomes.

Keywords: Total hip arthroplasty, Delta PF cup, FIT stem, LimaCorporate, HHS, WOMAC

*Correspondence: petrfulin@gmail.com

Orthopaedic Clinic 1st Faculty of Medicine Charles University and Motol

University Hospital, V Uvalu 84, 15006 Prague, Czech Republic

C C The Author(s). 2020 Open Access This article is licensed under a Creative Commons Attribution 4.0 International License, which permits use, sharing, adaptation, distribution and reproduction in any medium or format, as long as you give appropriate credit to the original author(s) and the source, provide a link to the Creative Commons licence, and indicate if changes were made. The images or other third party material in this article are included in the article's Creative Commons licence, unless indicated otherwise in a credit line to the material. If material is not included in the article's Creative Commons licence and your intended use is not permitted by statutory regulation or exceeds the permitted use, you will need to obtain permission directly from the copyright holder. To view a copy of this licence, visit http://creativecommons.org/licenses/by/4.0/. The Creative Commons Public Domain Dedication waiver (http://creativecommons.org/publicdomain/zero/1.0/) applies to the data made available in this article, unless otherwise stated in a credit line to the data. 


\section{Background}

Total hip replacement is the current proven mothod of treating many ailments of the hip joint. Worldwide, there is an increasing number of primary Total Hip Arthroplasties (THAs), along with THA revision surgeries as a consequence. As the indications for THA widen, patients that undergo surgery are more active and have longer lifespans. The lifetime of the implants is therefore a key parameter that the surgeon should take into account at the time of the primary THA. The surgeon should consider the expected level of activity of the patient; their biological and chronological age and associated diseases. The choice of articulating surfaces is a major factor in the expected lifetime of the implant. According to studies by Amanatullah, the ceramic-on-ceramic pairing of articulating surfaces offers the best friction properties [1]. The aim of this study is a clinico-radiographical assessment of the Delta PF-FIT (LimaCorporate, Italy) THA system with a ceramic-on-ceramic paring of articulating surfaces.

\section{Methods}

The Delta PF is a hemispherical cementless acetabular cup manufactured from Titanium alloy (Ti6Al4V), available in Italy from 2003 and in the Czech Republic from 2006. The surface finish of the cup has a coating of porous titanium and an additional layer of hydroxyapatite in order to stimulate bone growth and osseointegration of the cup [2]. The cup has equatorial retentive grooves for optimal press-fit effect (Fig. 1). The acetabular cup is available in sizes ranging from $44 \mathrm{~mm}$ to $66 \mathrm{~mm}$ in $2 \mathrm{~mm}$ increments. The inserts for the Delta PF cup have a patented peg at the rear for precise insertion into the metal cup. This prevents malposition of the insert, which may lead to splintering of the edge of the insert and possible early failure [2]. It also acts as a prevention for the concentration of forces in the ceramic material. The FIT stem is a titanium (Ti6Al4V), cementless anatomical prosthesis that has been on the market in the Czech Republic since 2005. The proximal part of the stem has porous titanium with a coating of hydroxyapatite for optimal implant integration. The stem was designed to offer maximum stability while respecting the morphology of the proximal femur (Fig. 1). The result of which is a physiological force transfer in the region of proximal femur similar to that of a healthy femur. The medial anatomical curve of the stem balances out the $9^{\circ}$ lateral curve in the base of the stem isthmus. Due to the asymmetrical shape of the implant, it ideally fills out the femoral canal and prevents abnormal force transfer to surrounding cortical bone; reducing the risk of stress shielding and thigh pain [2].

Between 2006 and 2012, we implanted 209 Delta PFFIT (LimaCorporate, Itálie) THAs with ceramic-onceramic (BIOLOX ${ }^{\circ}$ Delta) articulation surfaces in 174 patients at our clinic. Six patients (7 hips) died within the follow-up period and 4 patients (4 hips) were lost to follow-up. In one particular case, a revision was performed due to a periprosthetic fracture of the femur. Overall, 197 THAs were assessed both radiographically and clinically. $(\mathrm{F}=94, \mathrm{M}=103)$ in 163 patients (Fig. 2). The mean age at time of surgery was 57.8 years (range $24-81$ years SD $\pm 10,6)$. The left hip was operated in 103 cases and the right in 94 cases. The mean time of follow-up was 7.7 years (range $5.1-11.2$ years $\mathrm{SD} \pm 1,5$ ). The indications for THA are summarised in a table (Table 1 ).

Clinical evaluation was done with the use of the Harris Hip Score (HHS) [3] and Western Ontario and McMaster Universities Arthritis index (WOMAC Score) [4] pre- and post-operatively. The HHS evaluates the clinical and the functional result. The result is rated based on a set grading: Excellent (90-100 points), Good (80-89 points), fair (70-79 points) and poor (less than 70 points). The

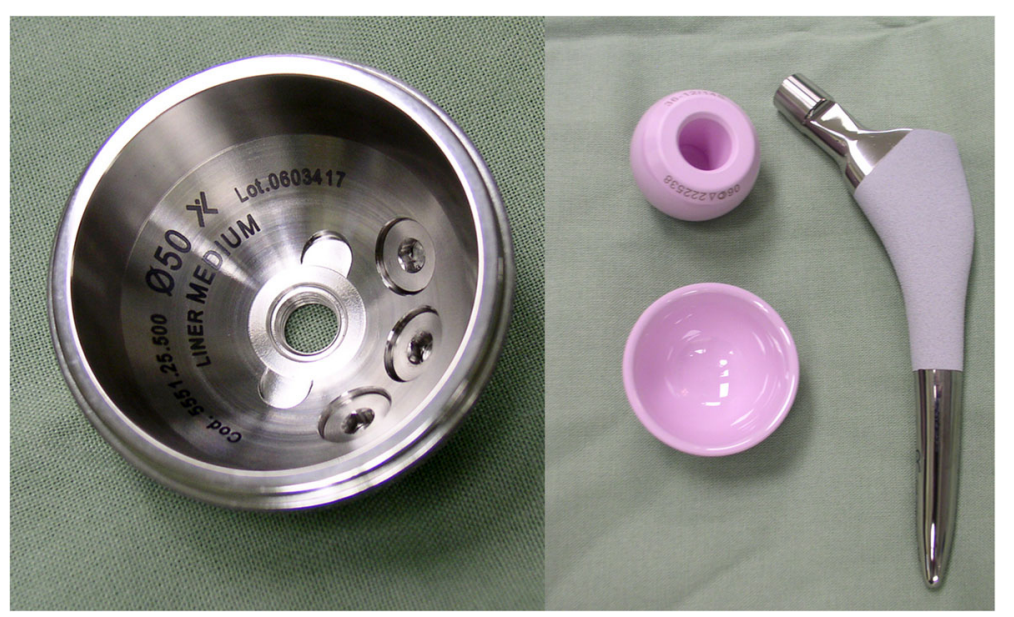

Fig. 1 Lima Implants (LimaCorporate). Delta PF Cup (left), FIT stem with ceramic insert and a BIOLOX® Delta head (right) 


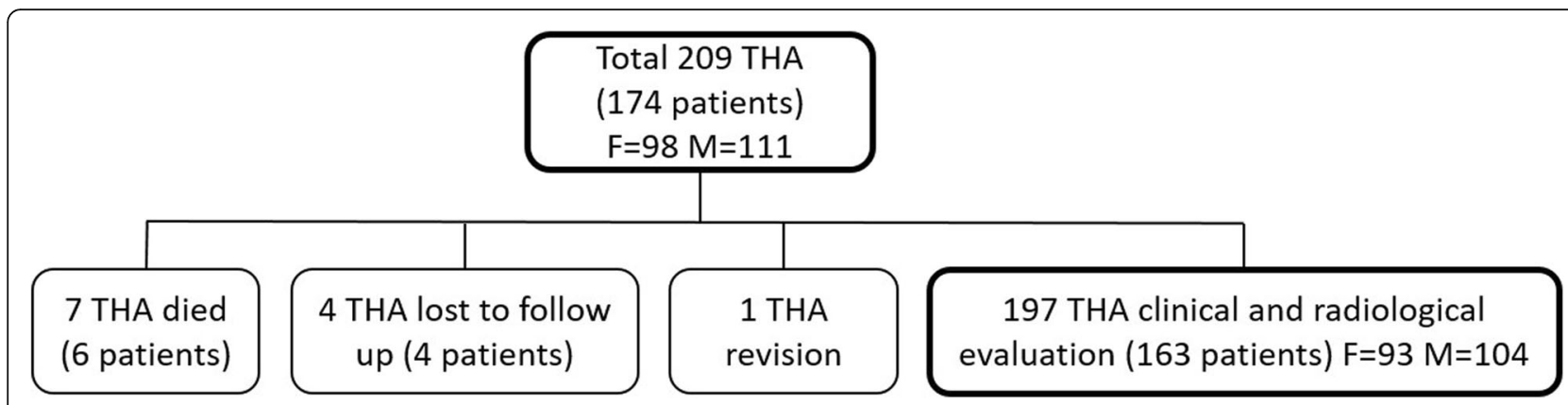

Fig. 2 Distribution of operated patients

WOMAC score evaluates pain, rigidity, other symptoms and function in daily life. (0-100 scale). The radiographical assessment evaluated the zone of translucency surrounding the femoral and acetabular components, cortical hypertrophy of the femur surrounding the stem, subsidence of the stem when compared to the postoperative radiograph, changes in cup orientation and the occurrence of periarticular ossifications.

All THAs were performed by 3 very experienced surgeons. In all cases the surgical approach was anterolateral with the patient in supine position. The cup was implanted by press-fit method in an inclination angle of $45^{\circ}$ and anteversion angle of $10-15^{\circ}$, a ceramic Biolox ${ }^{\circ}$ Delta insert was then inserted into the cup. The stem was implanted anatomically with the press-fit method. A Biolox Delta ceramic head was used in all cases.

The statistical evaluation included the calculation of mean, median andinterquartile range (IQR) values for selected variables. To compare the different hip replacement systems in the discussion, we also set up KaplanMeier survival analysis with $95 \%$ confidence intervals by using the Statistica 12 program (StatSoft, USA). We used scatterplot matrix graph and Pearson's and Spearmen's correlation coefficients for more detailed correlations of individual measurement values.

The study was approved by Ethic Committee of the University Hospital Motol (Reference No. EK-73/19). This is a retrospective study.

Table 1 Indications for THA

\begin{tabular}{lll}
\hline Indications & Number & \\
\hline Diagnosis & 153 & Percentage \\
\hline Arthritis & 23 & $77.7 \%$ \\
Postdysplastic arthritis & 10 & $11.7 \%$ \\
Femoral head necrosis & 4 & $5.1 \%$ \\
Rheumatic arthritis & 4 & $2.0 \%$ \\
Femoral neck fracture & 2 & $2.0 \%$ \\
Femoral nail failure & 1 & $1.0 \%$ \\
State after coxitis & & $0.5 \%$ \\
\hline
\end{tabular}

\section{Results}

Overall, 198 THAs out of the original 209 were evaluated (7 died, 4 lost to follow-up). One patient was revised due to a periprosthetic fracture of the femur (revision rate $0,51 \%$ ). We had no infectious complications. Therefore, 197 hips were assessed both clinically and radiographically.

\section{Clinical outcome assessment}

In 2 patients there were intraoperative complications; those being the formation of a fissure in the proximal femur during the impaction of the femoral stem, without affecting the primary stability of the implant. In both cases, spontaneous healing of the fracture occurred with a good final function of the implanted hip. Among postoperative complications, the most common was a limitation in the range of movement. A hip flexion contracture of up to $10^{\circ}$ occurred in 4 cases. A slight, intermittent pain was reported by 2 patients; one patient reported a slight pressure in the thigh during heavy loads. The average range of flexion was $104.16^{\circ}\left(70-130^{\circ}\right.$ range with a $10,0 \mathrm{IQR})$. The average range of abduction was $40.72^{\circ}$ $\left(30-50^{\circ}\right.$ range with a $\left.5,0 \mathrm{IQR}\right)$. The average range of external rotation was $28.95^{\circ}\left(0-40^{\circ}\right.$ range with a 0,0 IQR). The average range of internal rotation was $19,13^{\circ}$ $\left(0-35^{\circ}\right.$ range with $\left.2,0 \mathrm{IQR}\right)$ (Table 2). The mean HHS score was 97.59 points (61-100 range with a 3,0 IQR). 186 THAs were evaluated as excellent (90-100 points), 9 THAs rated as good (80-89 points), 1 THA was rated as fair (70-79 points) and 1 THA rated as poor (less than 70 points). Pre-operatively mean HSS was 51.21 (Table 3 )

Table 2 Statistical values (FU-follow up, FL- flexion, ABD abduction, ER - external rotation, IR- internal rotation, IQR interquartile range)

\begin{tabular}{lllllllll}
\hline & Age & FU & HHS & WOMAC & FL & ABD & ER & IR \\
\hline mean & 57.9 & 7.7 & 97.6 & 97.4 & 104.2 & 40.7 & 28.9 & 19.1 \\
median & 59.0 & 7.7 & 100.0 & 100.0 & 100.0 & 40.0 & 30.0 & 20.0 \\
5\% trimmed mean & 58.5 & 7.6 & 98.3 & 98.2 & 104.3 & 40.9 & 29.1 & 19.3 \\
IQR & 11.0 & 1.9 & 3.0 & 4.0 & 10.0 & 5.0 & 0.0 & 2.0 \\
\hline
\end{tabular}


and HHS increased in average by 46.38 points. The post-operatively mean WOMAC score was 97.38 points (65-100 range with a 4,0 IQR). Pre-operatively mean WOMAC was 50.12 (Table 3) and WOMAC increased in average by 47.26 points. In both cases, the rise in scores is statistically significant $(p \leq 0,05)$.

\section{Radiographic evaluation}

Radiolucent lines surrounding the acetabular or femoral components have not been observed in a single case in our cohort. There were also no changes in orientation or change of position of the acetabular cup. A slight femoral bone hypertrophy surrounding the stem (spot weld) was observed in one case. In two patients, a slight stem subsidence (approx. $5 \mathrm{~mm}$ ) of the femoral component was observed when compared to the postoperative radiograph. Both patients however have no functional limitation and are without problems. In both cases, the cause was most likely an incorrect selection of an undersized implant. A further 15 hips were noted as having an undrsized implant. Despite this, there has not been a single case of loosening; all patients are without problems. In these hips, we observed the remodelling of bone trabeculae with their integration onto the surface of the prosthesis (Fig. 3). In 11 patients, we observed a slight varus orientation of the stem on an antero-posterior radiograph without any corelating clinical problems. Wear of articulating surfaces was not been observed in a single case.

The occurrence of heterotopic ossifications was evaluated according to Brooker's classification [5]. Grade I was identified in 25 cases (12,7\%), grade II in 16 cases $(8,1 \%)$ and grade III in 5 cases $(2,5 \%)$. We did not observe any cases with Grade IV ossifications.

\section{Statistical analysis}

In our cohort of 198 THAs, only one revision was performed for a periprosthetic fracture. We documented an overall 99.49\% Kaplan-Meier survival with an average follow-up of 7.7 years with the FIT (LimaCorporate)

Table 3 Results of pre- and post-operatively clinical evaluation (SD - standard deviation)

\begin{tabular}{|c|c|c|c|c|c|c|c|c|}
\hline \multicolumn{9}{|l|}{ Results $(n=197)$} \\
\hline & \multicolumn{4}{|c|}{$\underline{\text { Preoperative }}$} & \multicolumn{4}{|c|}{ Postoperative } \\
\hline & Mean & Min & Max & SD & Mean & Min & Max & SD \\
\hline HSS & 51.21 & 28 & 73 & 4.92 & 97.59 & 61 & 100 & 5.13 \\
\hline WOMAC & 50.12 & 27 & 69 & 4.94 & 97.38 & 65 & 100 & 5.18 \\
\hline Flexion & 89.51 & 45 & 110 & 8.2 & 104.16 & 70 & 130 & 10.61 \\
\hline Abduction & 27.32 & 5 & 40 & 4.71 & 40.72 & 30 & 50 & 4.52 \\
\hline External rotation & 19.81 & 0 & 30 & 5.12 & 28.95 & 0 & 40 & 5.6 \\
\hline Internal rotation & 11.26 & 0 & 20 & 4.84 & 19.13 & 0 & 35 & 6.17 \\
\hline
\end{tabular}

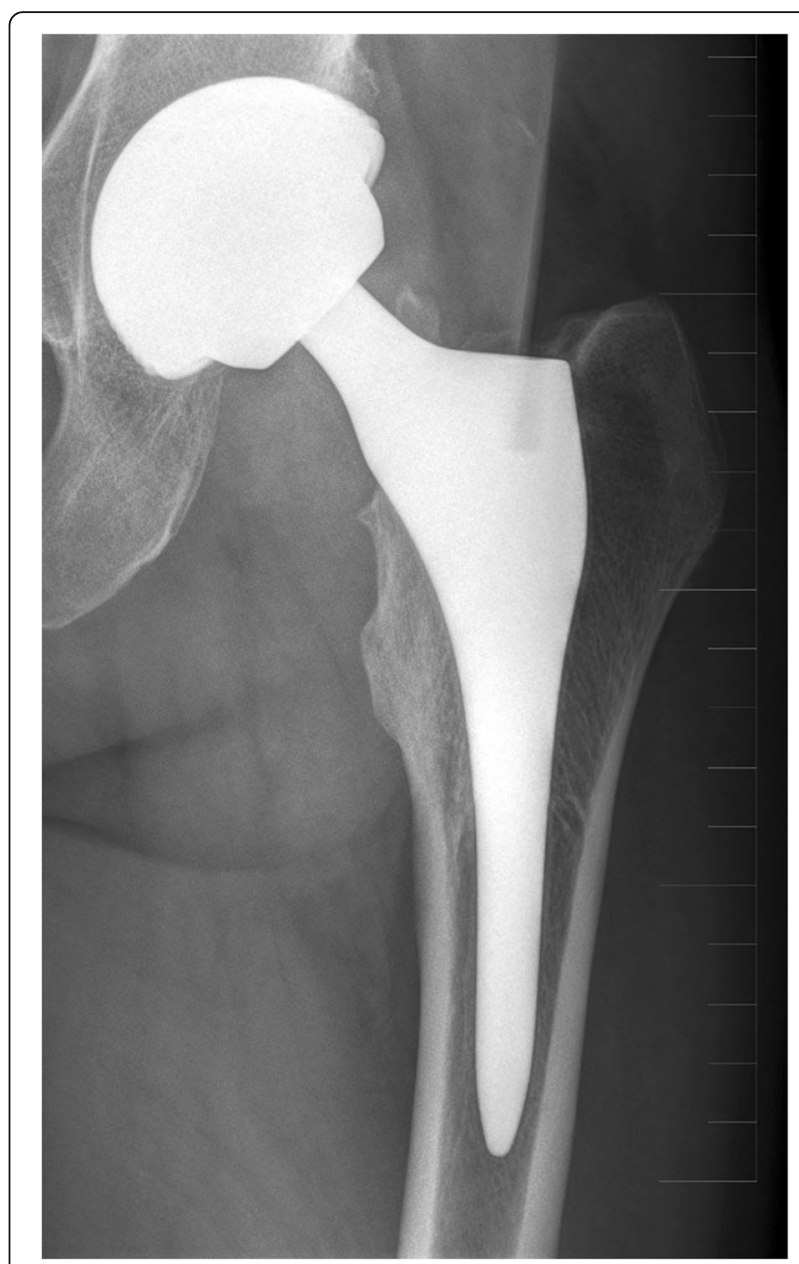

Fig. 3 Bone trabeculae remodelling with the integration into the hydroxyapatite surface of the implant

stem revision and any component revision as the endpoint. With the Delta PF (LimaCorporate) cup revision as the endpoint, the survival was $100 \%$. The statistical values for range of movement, HSS and WOMAC are shown in a table (Table 2). Correlations between variables were evaluated using a scatter matrix graph (Fig. 4). There is a significant correlation between the both of clinical evaluation (HHS and WOMAC (Fig. 5) (Pearson correlation coefficient (Pcc) 0,94, Spearman correlation coefficiant (Scc) 0,91)) as well as between clinical evaluation and range of motion. The highest correlation was observed between HHS and flexion (Pcc 0,6, Scc 0,55) and between WOMAC and flexion (Pcc 0,62, Scc 0,55) and also between internal rotation and abduction (Pcc 0 , 7 , Scc 0,7).

\section{Discussion}

Cementless THA are currently at the forefront of orthopaedic surgery. When examining publised studies, we have not found a clinical or radiographical assessment of 


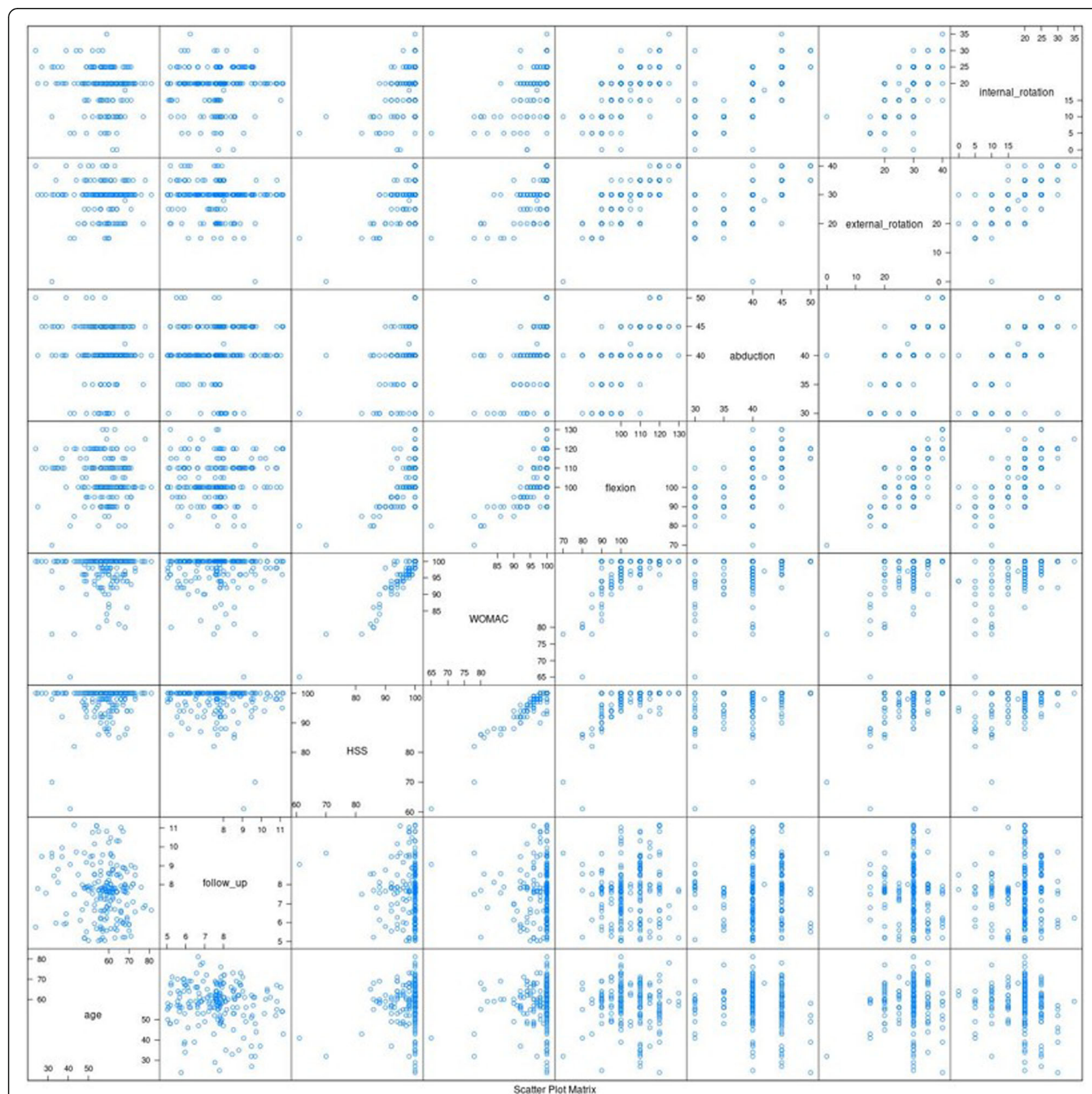

Fig. 4 Matrix of scatter plots. The highest correlations show the values closest to the linear ascending line. The lowest correlation followed by values with random scattering of values

this implant. Haverkamp et al [6] evaluated 222 patients with an implanted Delta PF (LimaCorporate) cup, with a follow-up period of 3-21 months. They showed a cumulative survivorship of $99.5 \%$ after 1 year. In the same study, they evaluated 250 Selexys cups (Mathys) with the same follow-up time and cumulative survivorship of $87.4 \%$ after 1 year with a $7.4 \%$ revision rate for aseptic loosening [6]. A fairly large rate of aseptic loosening of the Selexys cup (Mathys) was also shown by Ilchman et al [7], where they evaluated 115 THAs with a followup of 2-20 months.

In their study, Benazzo et al [8] evaluated the survival of the Modulus stem with a variable neck system (LimaCorporate). They found a $98.28 \%$ survivorship of the stem with an average HHS of 96.6 in 222 patients with an average follow-up of 5 years [8]. The increased role of anatomical and short stems is becoming more and more discussed. The results of a clinical evaluation of 

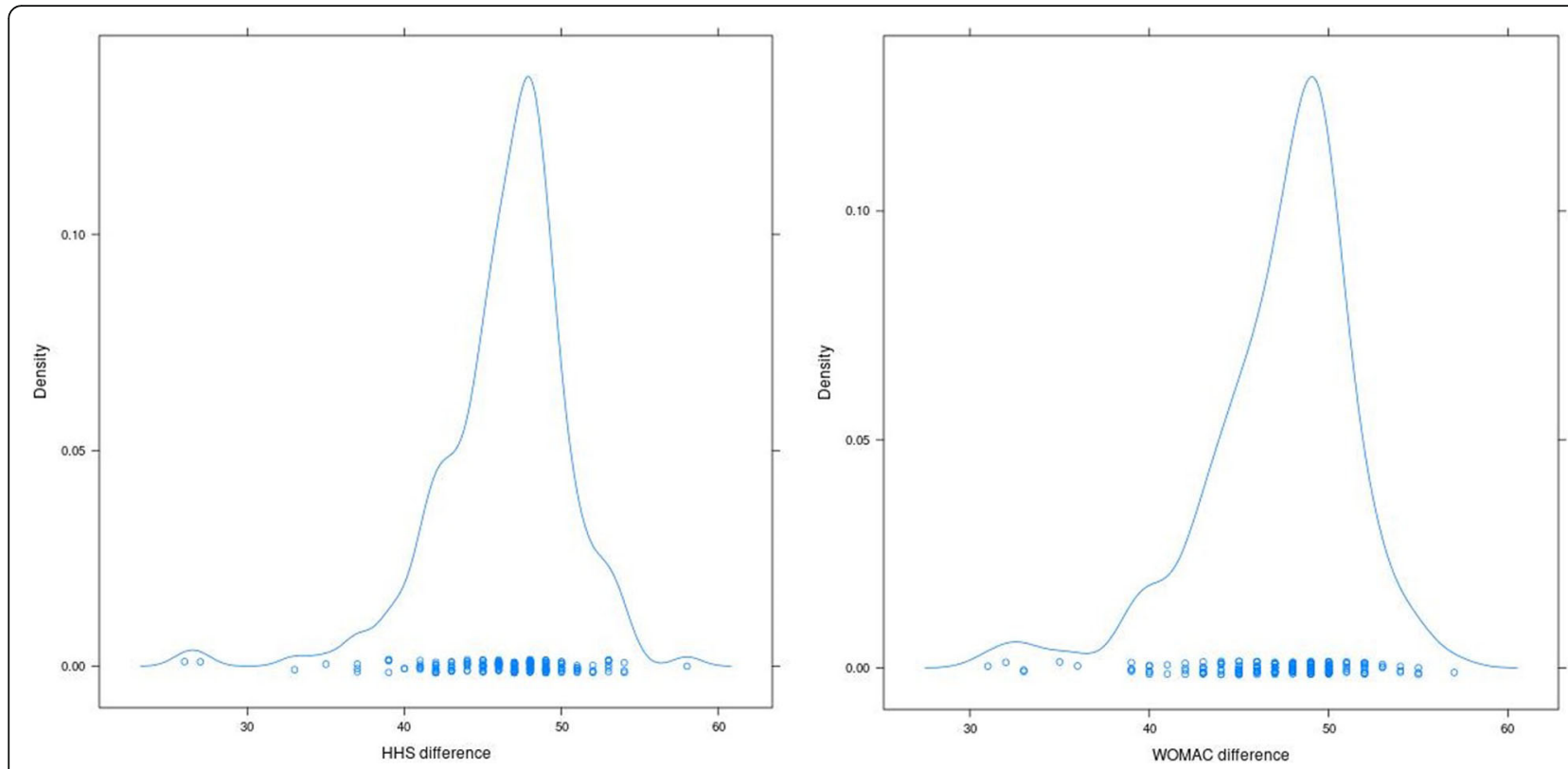

Fig. 5 The density of HHS difference (left side) and the density of WOMAC difference (right side)

the Fitmore (Zimmer) stem showed a HHS of 99.0 with a mean follow-up of 2 years and 3 revisions in the same period of time [9]. The evaluation of the PPF (proximal press-fit) (Biomet) stem in a cohort of 142 patients was published in 2016 [10]. Iori and Vigano showed a survivorship of $99.1 \%$ after 10 years. The PPF (Biomet) stem is a Zweymüller type of stem that has a long tradition and excellent results. There are many published studies evaluating the Alloclassic (Zimmer) stem. Both Zweymüller [11] and Delaunay [12] showed 98\% survival rates of the stem in their published studies. Similarly, studies published by Suckel and Grübl [13, 14] proved 98\% survival rates after 15 years. Likewise, other studies with a 9-11 year follow-up showed similar results (94\% - 97\%) when combined with the CSF cup (Zimmer) [15-18]. In their cohort of 154 patients with an implanted SL-plus stem (Smith \& Nephew), Studers et al found a 93.5\% survival rate with a mean HHS score of 92.7 and a 93.8 WOMAC [19].

In their group of 154 patients, Studers et al show a 93.5\% survival of the SL-plus stem after 10 years with a mean HHS of 92.7 and a WOMAC score of 93.8 [19]. The SL-plus/Bicon-plus system (Smith \& Nephew) was also evaluated by Kovoresis et al, where they published a survival of $97 \%$ after 11 years [20]. The Corail stem (DePuy) shows very good results. A $96.8 \%$ survival after 20.9 years in a cohort of 127 patients was shown by Vidalain [21]. Wangen et al published a survival of $96.9 \%$ of the Corail stem after 11 years with a mean HHS of 94 and a mean WOMAC score of 89 [22].

The amount of heterotopic ossifications occurring in our cohort might seem relatively large, however it is in concurrence with a number of previously published studies. When evaluating the Bicon/alloclassic SL system, Ottink et al showed a 71\% occurrence of heterotopic ossifications (grade I. 41\%, grade II. 20\%, grade III. 9\%, grade IV. 1\%) [23]. Won Sik Choy documented an 8.7\% occurrence of heterotopic ossifications with the Alumina ceramic head and Delta ceramic liner pairing [24]. In their study, White et al showed a $14.3 \%$ occurrence of ossifications (grade I. 5.5\%, grade II. 6.8\%, grade III. $1.7 \%$, grade IV. 0\%) [25]. White also mentioned that the risk factors for the occurrence of heterotopic ossification are the male gender and hypertrophic arthritis [25]. Kantak et al compared the influence of interoperative lavage on the occurrence of heterotopic ossifications. In a control group (surgical wound lavage of less than 1 litre), the incidence of heterotopic ossifications was $73.3 \%$ (66 out of 90 patients). Six of those patients had grade III or IV ossifications (6.7\%). In a group where a lavage of the surgical wound was performed with more than 3 litres of physiologic solution, the incidence of heterotopic ossifications was $41.5 \%$ (35 out of 85 patients), while no grade III or IV ossifications were observed [26].

\section{Conclusion}

Evaluation of the Delta-PF-FIT (LimaCorporate, Italy) THA system with the use of ceramic-on-ceramic BIOLOX ${ }^{\circ}$ Delta articulation surfaces shows very good outcomes. Clinical and radiographical evaluation confirms a marked subjective improvement in patient quality of life with a minimal number of complications. 


\section{Abbreviations}

THA: Total hip arthroplasty; HHS: Harris hip score; WOMAC: Western Ontario and McMaster Universities Arthritis index; SD: Standard deviation

\section{Acknowledgements}

Not Applicable

\section{Authors' contributions}

PF - Contributions to conception and design of study, acquisition, analysis and interpretation of data. Main author of article. JH - Contributions to acquisition, analysis and interpretation of data. DP - Contributions to analysis and interpretation of data and preparation of article. AS - Contributions to prepare design of study and preparation of article. All authors have read and approved the manuscript.

\section{Funding}

The study is supported by grant projects:

1) Agency for Healthcare Research Czech Republic (Ministry of Health Czech Republic)15-31269A - Participates in the financing of image documentation. 2) Ministry of Industry and Trade FV-30086 - Participates in the financing of manuscript translation.

3) Technology Agency of the Czech Republic TH-03010354 - Participates in technical operating costs.

4) The research program of the Charles University Q25/ LF1 / 2 - Participates in the purchase of office supplies.

5) Project (Ministry of Health) of the conceptual development research organizations 00064203 (Motol University Hospital) - Participates in the purchase of office supplies.

\section{Availability of data and materials}

All data and materials is available in xls format in case of interest (coressponding author - PF). The dataset supporting the conclusions of this article is available in xls format in case of interest it is possible send by email. The datasets are not available online.

\section{Ethics approval and consent to participate}

The study was approved by Ethic Committee of the University Hospital Motol (Reference No. EK-73/19). Written consent to the study was obtained from all participants.

\section{Consent for publication}

Manuscript not contain any person's data - not applicable.

\section{Competing interests}

The authors declare that they have no competing interests.

Received: 2 February 2019 Accepted: 31 March 2020

Published online: 19 May 2020

\section{References}

1. Amanatullah DF, Landa J, Strauss EJ, Garino JP, Kim SH, Di Cesare PE. Comparison of surgical outcomes and implant wear between ceramic ceramic and ceramic-polyethylene articulations in total hip arthroplasty. J Arthroplasty. 2011;26(6):72-7. https://doi.org/10.1016/j.arth.2011.04.032

2. LimaCorporate. https://www.limacorporate.com/medical/145/delta-pf. html?country=55. Accessed 24 Jan 2018.

3. Harris WH. Traumatic arthritis of the hip after dislocation and acetabular fractures: treatment by mold arthroplasty. An end-result study using a new method of result evaluation. J Bone Joint Surg Am. 1969;51(4):737-55.

4. Klassbo M, Larsson E, Mannevik E. Hip disability and osteoarthritis outcome score. An extension of the Western Ontario and McMaster universities osteoarthritis index. Scand J Rheumatol. 2003;32(1):46-51.

5. Brooker AF, Bowerman JW, Robinson RA, Riley LH Jr. Ectopic ossification following total hip replacement. Incidence and a method of classification. J Bone Joint Surg Am. 1973;55:1629-32.

6. Haverkamp D, Westerbos S, Campo MM, Boonstra RH, Rob Albers GH, van der Vis HM. Early loosening of a press-fit cup with ceramic-on-ceramic articulation: our early results. Arch Orthop Trauma Surg. 2013;133(12):1757-62.

7. Ilchmann T, Pannhorst C, Abächerli C, Clauss M. Early failure of a spherical pressfit cup. Annual meeting of the Swiss Society of Orthopaedics and Traumatology. 2010, St Gallen.
8. Benazzo F, Rossi SM, Cecconi D, Piovani L, Ravasi F. Mid-term results of an uncemented femoral stem with modular neck options. Hip Int. 2010;20(4): 427-33.

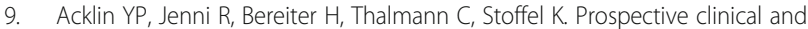
radiostereometric analysis of the Fitmore short-stem total hip arthroplasty. Arch Orthop Trauma Surg. 2016;136(2):277-84.

10. lori S, Viganò R. Good mid- to long-term THA outcomes with a modified cementless rectangular biconical stem design. Hip Int. 2016;26(4):380-5.

11. Zweymüller KA, Steindl M, Schwarzinger U. Good stability and minimal osteolysis with a biconical threaded cup at 10 years. Clin Orthop Relat Res. 2007:463(463):128-37.

12. Delaunay C, Kapandji Al. Survival analysis of cementless gritblasted titanium total hip arthroplasties. J Bone Joint Surg Br. 2001;83(3):408-13.

13. Suckel A, Geiger F, Kinzl L, Wulker N, Garbrecht M. Long-term results for the uncemented Zweymuller/Alloclassic hip endoprosthesis. A 15-year minimum follow-up of 320 hip operations. J Arthroplast. 2009;24(6):846-53.

14. Grübl A, Chiari C, Giurea A, et al. Cementless total hip arthroplasty with the rectangular titanium Zweymuller stem. A concise follow-up, at a minimum of fifteen years, of a previous report. J Bone Joint Surg Am. 2006;88(10): 2210-5.

15. Vervest TM, Anderson PG, Van Hout F, Wapstra FH, Louwerse RT, Koetsier JW. Ten to twelve-year results with the Zweymüller cementless total hip prosthesis. J Arthroplast. 2005;20(3):362-8.

16. Garcia-Cimbrelo E, Cruz-Pardos A, Madero R, Ortega-Andreu M. Total hip arthroplasty with use of the cementless Zweymuller Alloclassic system. A ten to thirteen-year follow-up study. J Bone Joint Surg Am. 2003;85-A(2): 296-303.

17. Pieringer $H$, Auersperg $V$, Griessler W, Böhler N. Long-term results with the cementless Alloclassic brand hip arthroplasty system. J Arthroplast. 2003; 18(3):321-8.

18. Perka C, Fischer U, Taylor WR, Matziolis G. Developmental hip dysplasia treated with total hip arthroplasty with a straight stem and a threaded cup. J Bone Joint Surg Am. 2004;86-A(2):312-9.

19. Studers $P$, Belajevs $D$, Jurkevics $V$, Likums $P$. Ten to fifteen-year clinical and radiographic follow-up with a third-generation cementless stem in a young patient population. Int Orthop. 2016;40:465-71. https://doi.org/10.1007/ s00264-015-2846-z.

20. Korovessis P, Repantis T, Zafiropoulos A. High medium-term survivorship and durability of Zweymüller-plus total hip arthroplasty. Arch Orthop Trauma Surg. 2011;131(5):603-11.

21. Vidalain JP. Twenty-year results of the cementless Corail stem. Int Orthop. 2011:35(2):189-94

22. Wangen $H$, Nordsletten $L$, Boldt JG, Fenstad AM, Beverland DE. The Corail stem as a reverse hybrid - survivorship and $x$-ray analysis at 10 years. Hip Int. 2017;27(4):354-60.

23. Ottink K, Barnaart L, Westerbeek R, van Kampen K, Bulstra S, van Jonbergen $H P$. Survival, clinical and radiological outcome of the Zweymüller SL/Biconplus total hip arthroplasty: a 15-year follow-up study. Hip Int. 2015;25(3): 204-8.

24. Choy WS, Cha YH, Jeon CY, Lee KS, Kim HY. A minimum ten years of followup of alumina head on Delta liner Total hip Arthroplasty. J Arthroplast. 2018, 33(2):470-6.

25. White PB, Ramkumar PN, Meftah M, Ghazi N, Ranawat AS, Ranawat CS. Incidence of heterotopic ossification following a multinodal pain protocol in total hip arthroplasty with the posterior approach. Orthopaedics. 2018;41(1): 92-7.

26. Kantak AP, Shah NN. Extensive surgical wound lavage reduces the incidence and severity of heterotopic ossification in primary total hip replacement: a study of 175 hip replacements. Hip and Pelvis. 2017;29(4):234-9.

\section{Publisher's Note}

Springer Nature remains neutral with regard to jurisdictional claims in published maps and institutional affiliations. 